\title{
Search for high-energy neutrino emission from hard X-ray AGN with IceCube
}

\section{The IceCube Collaboration}

(a complete list of authors can be found at the end of the proceedings)

E-mail: sreetama.goswami@icecube.wisc.edu

The IceCube Neutrino Observatory has detected high-energy astrophysical neutrinos in the TeV$\mathrm{PeV}$ range. These neutrinos have an isotropic distribution on the sky, and therefore, likely originate from extragalactic sources. Active Galactic Nuclei form a class of astronomical objects which are promising neutrino source candidates given their high electromagnetic luminosity and potential ability to accelerate cosmic rays up to energies greater than $10^{16} \mathrm{eV}$. Interactions of these cosmic rays within the AGN environment are expected to produce both neutrinos and pionic gamma rays. Some hadronic models of AGN emission suggest that such gamma rays can in turn interact with the dense photon fields of AGN and cascade down to hard X-rays and MeV gamma rays. We present an update on the IceCube stacking analysis searching for high-energy neutrinos from hard X-ray sources sampled from the Swift-BAT AGN Spectroscopic Survey.

Corresponding authors: Sreetama Goswami ${ }^{1 *}$, George C. Privon ${ }^{2}$, Marcos Santander ${ }^{1}$

${ }^{1}$ Department of Physics and Astronomy, University of Alabama, Tuscaloosa, AL 35487 0324, USA

${ }^{2}$ North American ALMA Science Center, National Radio Astronomy Observatory, Charlottesville, VA 22903-2475, USA

* Presenter

$37^{\text {th }}$ International Cosmic Ray Conference (ICRC 2021)

July 12 th $-23 r d, 2021$

Online - Berlin, Germany 


\section{Introduction}

IceCube has detected astrophysical neutrinos in the TeV-PeV range that show an isotropic angular distribution, and their dominant origin remains unidentified [1]. This points to extragalactic sources as a possible origin of the very-high-energy (VHE) neutrinos. Active Galactic Nuclei (AGN) is one such promising candidate source of VHE neutrinos. These are amongst the brightest objects in the universe owing to their high intrinsic luminosity. According to the unified model of AGN [2], these sources have a central supermassive black hole surrounded by an accretion disk and are sometimes accompanied by relativistic jets. Blazars are the AGN with the orientation of the jet lying close to our line of sight. AGN are powerful engines and capable of accelerating particles to very high energies. When the cosmic rays which are accelerated in the AGN environment interact with the ambient photon or matter field, they produce charged and neutral pions. The charged and neutral pions eventually decay to produce neutrinos and gamma rays, respectively, with comparable energies [3, 4]. Since gamma-ray emission at GeV-TeV energies has been observed from AGN, these are also likely emitters of VHE neutrinos.

An IceCube study from 2016 searched for neutrino point sources from the second Fermi-LAT AGN catalog (2LAC) [5, 6]. No significant excess was observed but upper limits were obtained for a contribution to the diffuse flux of cosmic neutrinos from blazars. However, a neutrino detected by IceCube on September 22, 2017, was found to be in temporal and spatial coincidence with a blazar, TXS 0506+056, that was in a flaring state [7]. This was the first evidence of a correlation between any astrophysical source and a high-energy neutrino. An examination of archival IceCube data found excess neutrinos from the direction of the same blazar between September 2014 and March 2015, when the blazar was not in a flaring state [8]. Another study conducted with 10 years of IceCube data, searched for point-like sources of neutrinos and found an excess of neutrinos from a Seyfert II galaxy, NGC 1068, at a significance level of $2.9 \sigma$ [9]. This provides further evidence in favor of AGN as possible neutrino sources.

There are various models that explain the production mechanism of high-energy neutrinos from AGN. Purely leptonic models can reproduce the spectral energy distribution (SED) of blazars without neutrino production, while purely hadronic models may fail to reproduce the SED completely (see Fig.1). According to some hybrid (leptohadronic) models, the TeV-PeV gamma rays produced alongside neutrinos can interact with the ambient photons in AGN and cascade down to hard X-rays (high energy $\mathrm{X}$-rays $\gtrsim 10 \mathrm{keV}$ ) or $\mathrm{MeV}$ gamma rays [10-12]. This creates a scenario where we can no longer observe the TeV-PeV gamma rays but possibly find signatures of hard $\mathrm{X}$-rays and $\mathrm{MeV}$ gamma rays. Hard X-rays could therefore be an important probe into the emission of neutrinos through hadronic processes.

\section{The catalog of hard X-ray sources}

We select the sources for our analysis from the hard X-ray AGN detected by Burst Alert Telescope (BAT) onboard the Niel Gehrels Swift gamma-ray burst observatory [14]. The SwiftBAT 70-Month hard X-ray Survey is the collection of objects observed by the BAT hard X-ray detector for a period of 70 months [15]. There are 1171 sources detected in the $>10 \mathrm{keV}$ band which are associated with 1210 counterparts. The counterparts were identified by an archival search 


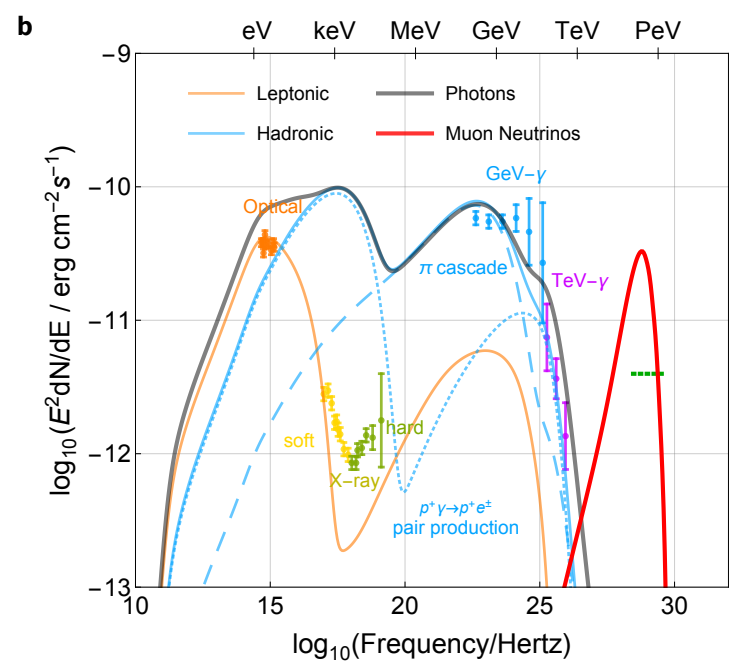

Figure 1: Spectral energy flux from TXS 0506+056 with different model fits. A simple hadronic model (in blue) used to fit the high energy peak overestimates the observed X-ray flux. In this analysis, the focus is on sources selected in the $14-195 \mathrm{keV}$ range (figure from [13]).

for point sources (within $\sim 15$ arcmin of BAT source position) in X-ray observations of Swift-XRT, Chandra, XMM-Newton, Suzaku and ASCA. If no counterpart was found, then a $10 \mathrm{ks}$ follow-up observation was carried out by Swift-XRT using the BAT source coordinate. If the sources still did not have an observation, from either the archives or Swift-XRT, then NED and SIMBAD were checked to find a likely counterpart.

The BAT AGN Spectroscopic Survey ${ }^{1}$, or the BASS catalog Data Release-1, is a selection of AGN sources from the 70-month BAT catalog [16]. It has 838 AGN sources, mostly nearby, $90 \%$ of them with a redshift below 0.2. Multi-wavelength physical properties of the sources have been determined by the analysis of the X-ray data along with the study of their available optical spectroscopic data [17].

The Swift-BAT survey presents a very uniform coverage of the sky. The BASS catalog is the most complete all-sky catalog of hard X-ray sources observed in the range of $14-195 \mathrm{keV}$ and includes soft X-ray spectra in the range of $0.3-10 \mathrm{keV}$ that are used to derive spectral parameters such as the column density. For two sources in the BASS catalog, there are no column density values since they do not have a soft X-ray observation in the $0.3-10 \mathrm{keV}$ range carried out by Swift-XRT, XMM-Newton-EPIC/PN, or Chandra-ACIS. These sources are SWIFT J1119.5+5132 and SWIFT J1313.6+3650A and they have not been included in our sample. Hence, we have 836 sources in our sample. A skymap of the BASS sources used in the study is shown in Fig.2. 105 of the sources in the catalog are classified as blazars and 731 as non-blazar AGN. Of the blazars, 26 are BL Lac objects, AGN whose optical spectrum is featureless, or with absorption lines that originate from the host galaxy and with weak and narrow emission lines; 53 are Flat Spectrum Radio Quasars (FSRQ), AGN with optical spectrum showing broad emission lines; and 26 blazars are of unidentified type.

${ }^{1}$ https://www.bass-survey.com/ 


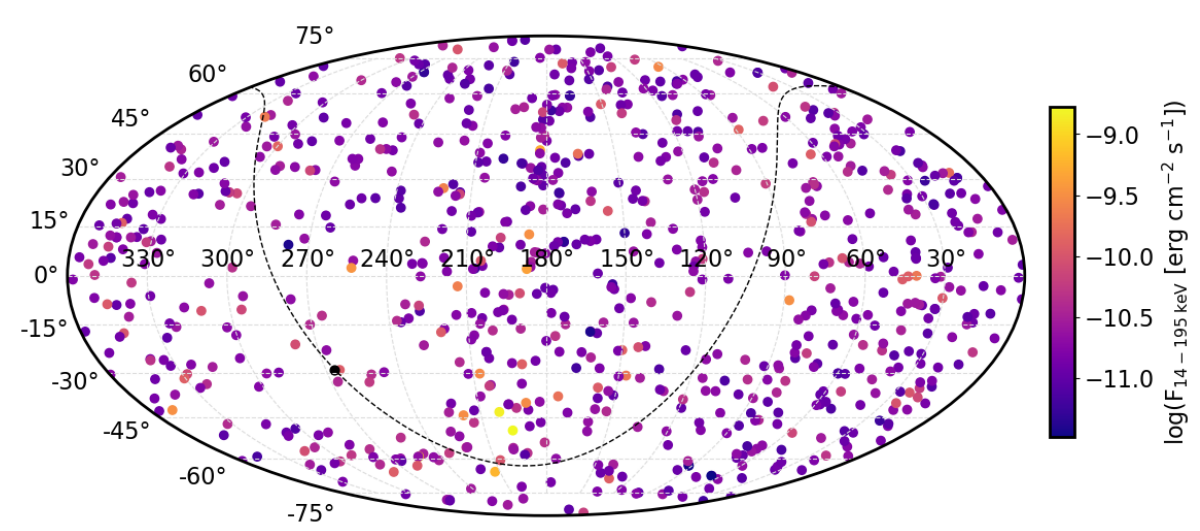

Figure 2: Skymap in equatorial coordinates showing the BASS sources and their intrinsic flux in the hard X-ray band of energy $14-195 \mathrm{keV}$. The dashed black line shows the Galactic plane (figure from [18]).

\subsection{Possible overlap with other studies}

AGN as a source class has been explored in an IceCube study that searched for neutrino emission from gamma-ray sources in the Fermi 2LAC catalog and has found no significant correlation [5]. Several hard X-ray sources in the BASS catalog also exhibit gamma-ray emission (see Fig.3). We have evaluated the possible overlap with this previous IceCube study.

A search was made for Fermi sources in the 4FGL catalog [19] located within a $0.2^{\circ}$ circular region of interest (ROI) around the BASS sources. Since most of the gamma-ray sources in 4FGL have a lower angular uncertainty than $0.2^{\circ}$, it is a conservative choice. 84 or $10 \%$ of the BASS sources have a 4FGL source within the ROI and 61 or 7\% of the BASS sources are consistent with a 4FGL position considering its angular uncertainty. Most of the high-luminosity sources (most often blazars) are found in both catalogs. We conclude that the number of sources common with previous studies is not predominant. Thus, this work introduces a novel and unexplored class of neutrino source candidates.

\section{Analysis outline}

\subsection{IceCube dataset}

IceCube is a cubic-kilometer neutrino detector installed in the ice at the geographic South Pole [20] between depths of $1450 \mathrm{~m}$ and $2450 \mathrm{~m}$, completed in 2010. It contains 5160 digital optical modules (DOM) that detect Cherenkov radiation emitted by charged particles produced in the interactions of neutrinos in the surrounding ice or the nearby bedrock. This radiation is then used in the reconstruction of the direction, energy, and flavor of the neutrinos.

The diffuse flux of VHE neutrinos observed by IceCube includes neutrino-interaction events with different topologies such as cascades and tracks [21]. The tracks, ascribed to $v_{\mu}$ and anti- $v_{\mu}$, have typical angular resolutions of $<1^{\circ}$. The main source of background for the cosmic neutrino flux consists of atmospheric muons and atmospheric neutrinos, both produced in cosmic-ray interactions in the Earth's atmosphere [22, 23]. 


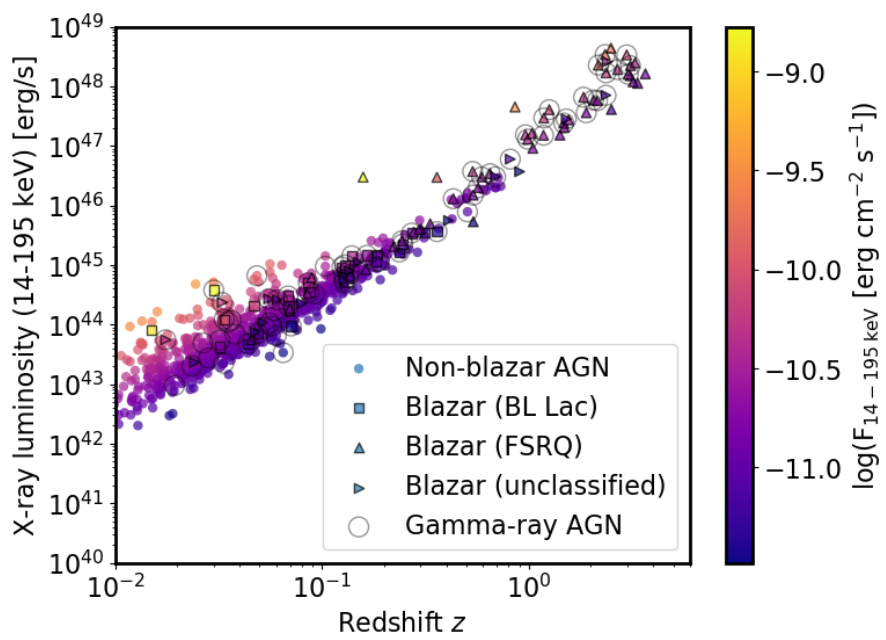

Figure 3: The figure shows the hard X-ray luminosity of the different classes of AGN in the BASS catalog as a function of their redshift. The color represents the intrinsic flux of the sources in the energy range of $14-195 \mathrm{keV}$. AGN sources with likely counterparts in the Fermi-LAT 4FGL catalog are circled in gray (figure from [18]).

For this analysis, the IceCube dataset used is the gamma-ray follow-up (GFU) sample [24]. It solely contains muon neutrino tracks with an all-sky coverage that have been reprocessed offline for better sensitivity. 7.5 years of the GFU sample from the complete 86-string IceCube configuration between 2011-2018 have been used.

\subsection{Likelihood approach}

The analysis involves a stacked search for point sources using a time-integrated unbinned maximum-likelihood method $[25,26]$. The likelihood function is given by:

$$
\mathcal{L}\left(n_{s}, \gamma\right)=\prod_{i}^{N}\left(\frac{n_{s}}{N} \mathcal{S}_{i}\left(\vec{x}_{i}, \sigma_{i}, E_{i}, \gamma\right)+\left(1-\frac{n_{s}}{N}\right) \mathcal{B}_{i}\left(\vec{x}_{i}, E_{i}\right)\right) .
$$

$\mathcal{S}$ and $\mathcal{B}$ represent the source and background probability density functions, respectively. Here, it is assumed that there is $n_{s}$ number of signal neutrinos that follow a power-law spectrum $E^{-\gamma}$. The likelihood is evaluated using data events with reconstructed direction and energy given by $\vec{x}_{i}$ and $E_{i}$, respectively, and $\sigma_{i}$ is the reconstructed directional uncertainty of each event. There is a total of $N$ neutrino events.

For a stacking analysis, the source PDF is a collective contribution from all the sources combined into one term. Each source term carries two kinds of weights; $\omega^{k}$, a theoretical weight depending on observed physical properties of the sources, and $R^{k}\left(\delta_{k}, \gamma\right)$, a detector weight which is proportional to the effective area of the IceCube detector for a source at a declination $\delta$ with a spectral index $\gamma$. The stacked-source PDF for $M$ sources is given by:

$$
\mathcal{S}_{i, \text { Stacked }}=\frac{\sum_{k=1}^{M} \omega^{k} R^{k}\left(\delta_{k}, \gamma\right) \cdot \mathcal{S}_{i}^{k}\left(\vec{x}_{i}, \vec{x}_{k}, \sigma_{i}, E_{i}, \gamma\right)}{\sum_{k=1}^{M} \omega^{k} R^{k}\left(\delta_{k}, \gamma\right)} .
$$


Then, the likelihood $\mathcal{L}$ is maximized resulting in the best-fit values of the parameters, $n_{s}=\hat{n}_{s}$ and $\gamma=\hat{\gamma}$. These values are used to calculate a Test Statistic $(\mathcal{T S})$ value according to the equation:

$$
\mathcal{T S}=-2 \operatorname{sgn}\left(\hat{n}_{s}\right) \log \left(\frac{\mathcal{L}\left(n_{s}=0\right)}{\mathcal{L}\left(n_{s}=\hat{n}_{s}, \gamma=\hat{\gamma}\right)}\right) .
$$

The larger the value of the $\mathcal{T S}$, the less compatible is the data with a background-only hypothesis.

The background-only distribution of TS is evaluated by randomizing the coordinates of the data. Only the right ascension, RA, of neutrino events are scrambled to preserve the declination distribution of the events. Each scrambling process is a trial. To test the performance of the analysis, pseudo signals generated from Monte Carlo simulations following a power-law spectrum are injected into the data in the position of the BASS sources.

We plan to test different hypotheses with our analysis. Currently, we have only considered the hypothesis outlined below, and we present the other hypotheses in the outlook of the proceedings. Hypothesis I: In this part, we use all the 836 AGN from the BASS DR-1 catalog to perform the analysis. Weighting Scheme:

1. Flux weights: This is proportional to the intrinsic flux in the hard X-ray range of $14-195$ $\mathrm{keV}$ of the AGN sources. This is selective towards the sources with higher flux in this energy range.

2. Equal weights: This is an unbiased weight that treats all sources in the catalog the same.

\section{Current status: Sensitivity and Discovery Potential}

The sensitivity and the $5 \sigma$ discovery potential are computed to give a sense of the power of the analysis performed. The sensitivity is defined as the flux required so that $90 \%$ of injected signal trials have a value of TS higher than in $50 \%$ of the background-only trials. The $5 \sigma$ discovery potential is the flux required so that $50 \%$ of the time this flux will produce a result inconsistent with the background-only hypothesis at a significance of $5 \sigma$. Fig. 4 and Fig. 5 show the preliminary estimates for the sensitivity and the $5 \sigma$ discovery potential under Hypothesis I.

\section{Outlook}

In future work, we will perform parts II and III of the analysis as described below.

Hypothesis II: We will search for a correlation between the luminosity of the sources and their neutrino emission by performing analyses on two of the following sub-classes of AGN in the catalog: Blazars, the AGN with high luminosity, and non-blazars, the AGN with relatively lower luminosity. The categorization follows the classification presented in the Swift-BAT catalog. In this analysis, only Flux weights will be applied and the results from each of the categories will be compared.

Hypothesis III: X-rays are an important tracer of AGN properties such as the neutral hydrogen column density $\left(N_{\mathrm{H}}\right)$ which is a measure of the obscuring material along the line of sight. Obscuring matter can act as a target for the high-energy photons such as hard X-rays, partially attenuating and 

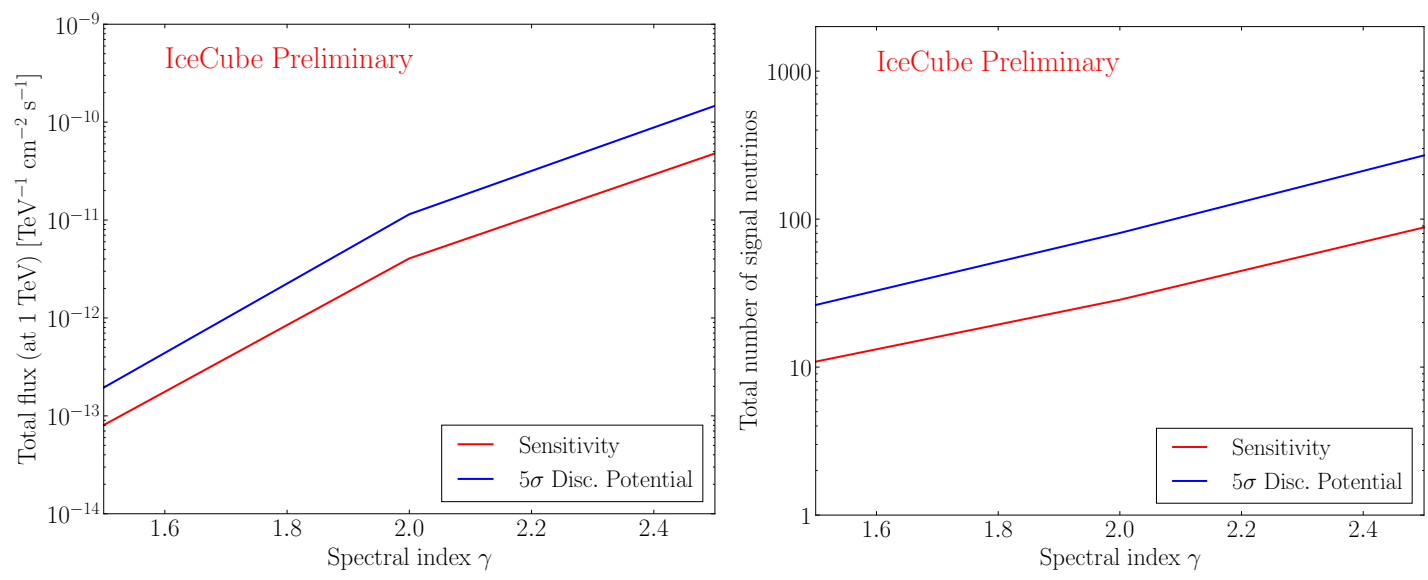

Figure 4: Plots showing the stacked flux at the normalization energy $1 \mathrm{TeV}$ (left) and the number of signal neutrinos (right) as a function of the spectral index with the sensitivity and the $5 \sigma$ discovery potential estimates, with Flux weights.
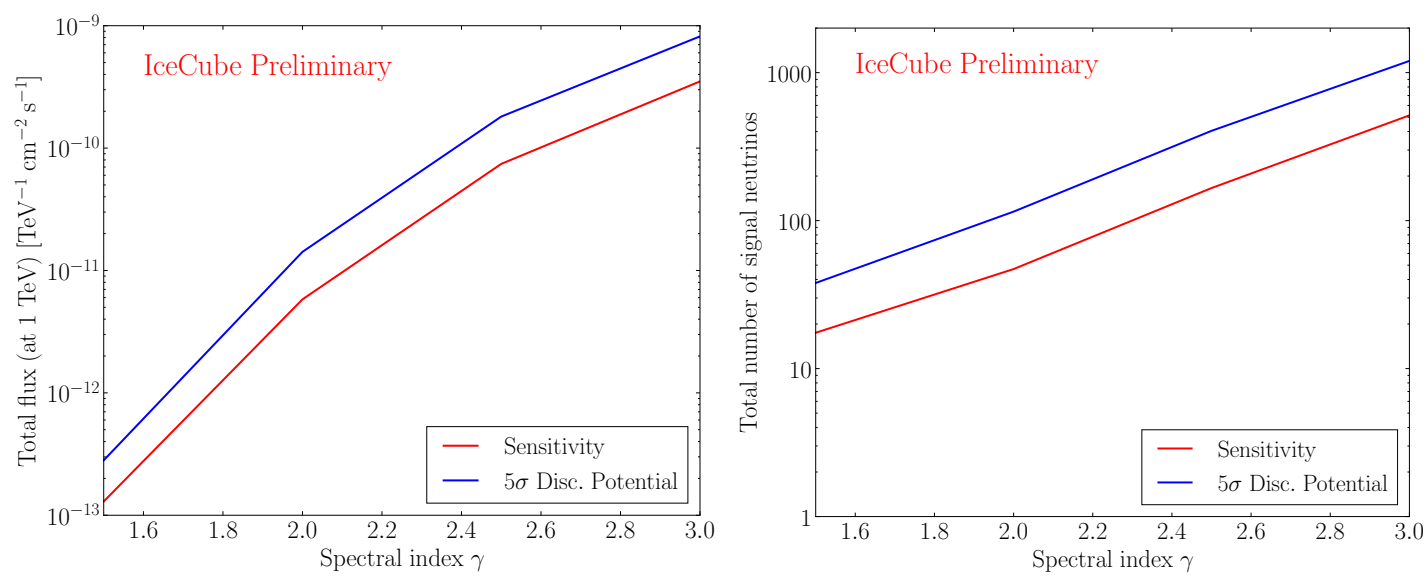

Figure 5: Plots showing the stacked flux at the normalization energy $1 \mathrm{TeV}$ (left) and the number of signal neutrinos (right) as a function of the spectral index with the sensitivity and the $5 \sigma$ discovery potential estimates, with Equal weights.

scattering these via Compton scattering and photoelectric absorption, and also affect the hadronic production of neutrinos. We will search for a correlation between the obscuring matter and the neutrino emission by dividing the sources in the catalog into three categories following the classification given in [17]. Unobscured sources are the sources with a column density, $N_{\mathrm{H}}<10^{22}$ $\mathrm{cm}^{-2}$; Obscured sources are the sources with a column density, $10^{22} \mathrm{~cm}^{-2} \leq N_{\mathrm{H}}<10^{24} \mathrm{~cm}^{-2}$; and Compton-thick sources are the sources with a column density, $N_{\mathrm{H}} \geq 10^{24} \mathrm{~cm}^{-2}$. Results from the analysis of each of these categories will be compared. In this approach only Flux weights will be applied.

From our analysis, we will either report the detection of a source and the associated neutrino flux or attain upper limits that constrain the contribution of AGN as a source class towards the diffuse flux of neutrinos observed by IceCube. 


\section{References}

[1] IceCube Collaboration, M. G. Aartsen et al. Science 342 (2013) 1242856.

[2] H. Netzer Annual Review of Astronomy and Astrophysics 53 no. 1, (Aug, 2015) 365-408.

[3] S. R. Kelner et al. Phys. Rev. D74 (2006) 034018. [Erratum: Phys. Rev.D79,039901(2009)].

[4] S. R. Kelner et al. Phys. Rev. D78 (2008) 034013. [Erratum: Phys. Rev.D82,099901(2010)].

[5] IceCube Collaboration, M. G. Aartsen et al. Astrophys. J. 835 no. 1, (2017) 45.

[6] IceCube Collaboration, M. Huber PoS ICRC2019 (2020) 916.

[7] IceCube, Fermi-LAT, MAGIC, AGILE, ASAS-SN, HAWC, H.E.S.S., INTEGRAL, Kanata, Kiso, Kapteyn, Liverpool Telescope, Subaru, Swift NuSTAR, VERITAS, VLA/17B-403

Collaboration, M. G. Aartsen et al. Science 361 no. 6398, (2018) eaat1378.

[8] IceCube Collaboration, M. G. Aartsen et al. Science 361 no. 6398, (2018) 147-151.

[9] IceCube Collaboration, M. G. Aartsen et al. Phys. Rev. Lett. 124 no. 5, (2020) 051103.

[10] M. Petropoulou et al. Mon. Not. Roy. Astron. Soc. 448 no. 3, (2015) 2412-2429.

[11] M. Petropoulou and A. Mastichiadis "Mon. Not. Roy. Astron. Soc." 447 (Feb, 2015) 36-48.

[12] K. Murase, F. Oikonomou, and M. Petropoulou Astrophys. J. 865 no. 2, (2018) 124.

[13] S. Gao, A. Fedynitch, W. Winter, and M. Pohl Nature Astron. 3 no. 1, (2019) 88-92.

[14] S. D. Barthelmy et al. Space Sci. Rev. 120 (2005) 143.

[15] W. H. Baumgartner et al. The Astrophysical Journal Supplement Series 207 no. 2, (Jul, 2013$) 19$.

[16] M. Koss et al. Astrophys. J. 850 no. 1, (2017) 74.

[17] C. Ricci et al. The Astrophysical Journal Supplement Series 233 no. 2, (Dec, 2017) 17.

[18] IceCube Collaboration PoS ICRC2019 (2019) 1002.

[19] S. Abdollahi et al. The Astrophysical Journal Supplement Series 247 no. 1, (Mar, 2020) 33.

[20] IceCube Collaboration, M. G. Aartsen et al. JINST 12 no. 03, (2017) P03012.

[21] Science 342 no. 6161, (Nov, 2013) 1242856-1242856.

[22] IceCube Collaboration, M. G. Aartsen et al. Phys. Rev. D 91 (Jun, 2015) 122004.

[23] IceCube Collaboration, M. Aartsen et al. Astroparticle Physics 78 (May, 2016) 1-27.

[24] IceCube Collaboration, M. Aartsen et al. Astroparticle Physics 92 (Jun, 2017) 30-41.

[25] A. Achterberg et al. Astroparticle Physics 26 no. 4-5, (Nov, 2006) 282-300.

[26] J. Braun et al. Astroparticle Physics 29 no. 4, (May, 2008) 299-305. 


\section{Full Author List: IceCube Collaboration}

R. Abbasi ${ }^{17}$, M. Ackermann ${ }^{59}$, J. Adams ${ }^{18}$, J. A. Aguilar ${ }^{12}$, M. Ahlers ${ }^{22}$, M. Ahrens ${ }^{50}$, C. Alispach ${ }^{28}$, A. A. Alves Jr. ${ }^{31}$, N. M. $\mathrm{Amin}^{42}$, R. An ${ }^{14}$, K. Andeen ${ }^{40}$, T. Anderson ${ }^{56}$, G. Anton ${ }^{26}$, C. Argüelles ${ }^{14}$, Y. Ashida ${ }^{38}$, S. Axani ${ }^{15}$, X. Bai ${ }^{46}$, A. Balagopal V. ${ }^{38}$, A. Barbano ${ }^{28}$, S. W. Barwick ${ }^{30}$, B. Bastian ${ }^{59}$, V. Basu ${ }^{38}$, S. Baur ${ }^{12}$, R. Bay ${ }^{8}$, J. J. Beatty ${ }^{20,21}$, K.-H. Becker ${ }^{58}$, J. Becker Tjus ${ }^{11}$, C. Bellenghi $^{27}$, S. BenZvi ${ }^{48}$, D. Berley ${ }^{19}$, E. Bernardini ${ }^{59}, 60$, D. Z. Besson ${ }^{34,61}$, G. Binder ${ }^{8}$,, D. Bindig ${ }^{58}$, E. Blaufuss ${ }^{19}$, S. Blot ${ }^{59}$, M. Boddenberg ${ }^{1}$, F. Bontempo ${ }^{31}$, J. Borowka ${ }^{1}$, S. Böser ${ }^{39}$, O. Botner ${ }^{57}$, J. Böttcher ${ }^{1}$, E. Bourbeau ${ }^{22}$, F. Bradascio ${ }^{59}$, J. Braun ${ }^{38}$, S. Bron $^{28}$, J. Brostean-Kaiser ${ }^{59}$, S. Browne ${ }^{32}$, A. Burgman ${ }^{57}$, R. T. Burley ${ }^{2}$, R. S. Busse ${ }^{41}$, M. A. Campana ${ }^{45}$, E. G. Carnie-Bronca ${ }^{2}$, C. Chen ${ }^{6}$, D. Chirkin ${ }^{38}$, K. Choi ${ }^{52}$, B. A. Clark ${ }^{24}$, K. Clark ${ }^{33}$, L. Classen ${ }^{41}$, A. Coleman ${ }^{42}$, G. H. Collin ${ }^{15}$, J. M. Conrad ${ }^{15}$, P. Coppin $^{13}$, P. Correa ${ }^{13}$, D. F. Cowen ${ }^{55}, 56$, R. Cross $^{48}$, C. Dappen ${ }^{1}$, P. Dave ${ }^{6}$, C. De Clercq ${ }^{13}$, J. J. DeLaunay ${ }^{56}$, H. Dembinski ${ }^{42}$, K. Deoskar $^{50}$, S. De Ridder ${ }^{29}$, A. Desai ${ }^{38}$, P. Desiati ${ }^{38}$, K. D. de Vries ${ }^{13}$, G. de Wasseige ${ }^{13}$, M. de With ${ }^{10}$, T. DeYoung ${ }^{24}$, S. Dharani $^{1}$, A. Diaz ${ }^{15}$, J. C. Díaz-Vélez ${ }^{38}$, M. Dittmer ${ }^{41}$, H. Dujmovic ${ }^{31}$, M. Dunkman ${ }^{56}$, M. A. DuVernois ${ }^{38}$, E. Dvorak ${ }^{46}$, T. Ehrhardt ${ }^{39}$, P. Eller $^{27}$, R. Engel ${ }^{31,32}$, H. Erpenbeck ${ }^{1}$, J. Evans ${ }^{19}$, P. A. Evenson ${ }^{42}$, K. L. Fan ${ }^{19}$, A. R. Fazely ${ }^{7}$, S. Fiedlschuster ${ }^{26}$, A. T. Fienberg ${ }^{56}$, K. Filimonov ${ }^{8}$, C. Finley ${ }^{50}$, L. Fischer ${ }^{59}$, D. Fox ${ }^{55}$, A. Franckowiak ${ }^{11,59}$, E. Friedman ${ }^{19}$, A. Fritz ${ }^{39}$, P. Fürst ${ }^{1}$, T. K. Gaisser ${ }^{42}$, J. Gallagher $^{37}$, E. Ganster ${ }^{1}$, A. Garcia ${ }^{14}$, S. Garrappa ${ }^{59}$, L. Gerhardt ${ }^{9}$, A. Ghadimi ${ }^{54}$, C. Glaser ${ }^{57}$, T. Glauch ${ }^{27}$, T. Glüsenkamp ${ }^{26}$, A. Goldschmidt $^{9}$, J. G. Gonzalez ${ }^{42}$, S. Goswami ${ }^{54}$, D. Grant ${ }^{24}$, T. Grégoire ${ }^{56}$, S. Griswold ${ }^{48}$, M. Gündüz ${ }^{11}$, C. Günther ${ }^{1}$, C. Haack ${ }^{27}$, A. Hallgren ${ }^{57}$, R. Halliday ${ }^{24}$, L. Halve ${ }^{1}$, F. Halzen ${ }^{38}$, M. Ha Minh ${ }^{27}$, K. Hanson ${ }^{38}$, J. Hardin ${ }^{38}$, A. A. Harnisch ${ }^{24}$, A. Haungs ${ }^{31}$, S. Hauser $^{1}$, D. Hebecker ${ }^{10}$, K. Helbing ${ }^{58}$, F. Henningsen ${ }^{27}$, E. C. Hettinger ${ }^{24}$, S. Hickford ${ }^{58}$, J. Hignight ${ }^{25}$, C. Hill ${ }^{16}$, G. C. Hill ${ }^{2}$, K. D.

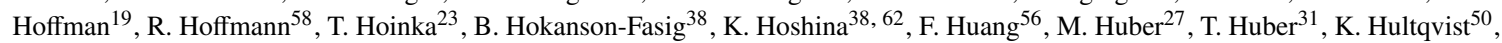
M. Hünnefeld ${ }^{23}$, R. Hussain ${ }^{38}$, S. In ${ }^{52}$, N. Iovine ${ }^{12}$, A. Ishihara ${ }^{16}$, M. Jansson ${ }^{50}$, G. S. Japaridze ${ }^{5}$, M. Jeong ${ }^{52}$, B. J. P. Jones ${ }^{4}$, D. Kang ${ }^{31}$, W. Kang ${ }^{52}$, X. Kang ${ }^{45}$, A. Kappes ${ }^{41}$, D. Kappesser ${ }^{39}$, T. Karg ${ }^{59}$, M. Karl ${ }^{27}$, A. Karle ${ }^{38}$, U. Katz ${ }^{26}$, M. Kauer ${ }^{38}$, M. Kellermann ${ }^{1}$, J. L. Kelley $^{38}$, A. Kheirandish ${ }^{56}$, K. Kin ${ }^{16}$, T. Kintscher ${ }^{59}$, J. Kiryluk ${ }^{51}$, S. R. Klein ${ }^{8,9}$, R. Koirala ${ }^{42}$, H. Kolanoski ${ }^{10}$, T. Kontrimas ${ }^{27}$, L. $\mathrm{Köpke}^{39}$, C. Kopper ${ }^{24}$, S. Kopper ${ }^{54}$, D. J. Koskinen ${ }^{22}$, P. Koundal ${ }^{31}$, M. Kovacevich ${ }^{45}$, M. Kowalski ${ }^{10,59}$, T. Kozynets ${ }^{22}$, E. Kun ${ }^{11}$, N. Kurahashi ${ }^{45}$, N. Lad ${ }^{59}$, C. Lagunas Gualda ${ }^{59}$, J. L. Lanfranchi ${ }^{56}$, M. J. Larson ${ }^{19}$, F. Lauber ${ }^{58}$, J. P. Lazar ${ }^{14,38}$, J. W. Lee ${ }^{52}$, K. Leonard $^{38}$, A. Leszczyńska ${ }^{32}$, Y. Li ${ }^{56}$, M. Lincetto ${ }^{11}$, Q. R. Liu ${ }^{38}$, M. Liubarska ${ }^{25}$, E. Lohfink ${ }^{39}$, C. J. Lozano Mariscal ${ }^{41}$, L. Lu ${ }^{38}$, F. Lucarelli ${ }^{28}$, A. Ludwig ${ }^{24}, 35$, W. Luszczak ${ }^{38}$, Y. Lyu ${ }^{8,9}$, W. Y. Ma ${ }^{59}$, J. Madsen ${ }^{38}$, K. B. M. Mahn ${ }^{24}$, Y. Makino ${ }^{38}$, S. Mancina ${ }^{38}$,

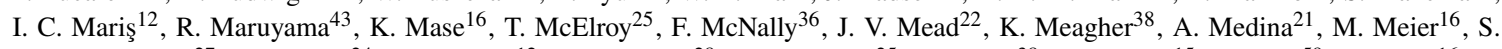
Meighen-Berger ${ }^{27}$, J. Micallef ${ }^{24}$, D. Mockler ${ }^{12}$, T. Montaruli ${ }^{28}$, R. W. Moore ${ }^{25}$, R. Morse ${ }^{38}$, M. Moulai ${ }^{15}$, R. Naab ${ }^{59}$, R. Nagai ${ }^{16}$, U. Naumann $^{58}$, J. Necker ${ }^{59}$, L. V. Nguyễn ${ }^{24}$, H. Niederhausen ${ }^{27}$, M. U. Nisa ${ }^{24}$, S. C. Nowicki ${ }^{24}$, D. R. Nygren ${ }^{9}$, A. Obertacke Pollmann ${ }^{58}$, M. Oehler ${ }^{31}$, A. Olivas ${ }^{19}$, E. O'Sullivan ${ }^{57}$, H. Pandya ${ }^{42}$, D. V. Pankova ${ }^{56}$, N. Park ${ }^{33}$, G. K. Parker ${ }^{4}$, E. N. Paudel ${ }^{42}$, L. Paul ${ }^{40}$, C. Pérez de los $\operatorname{Heros}^{57}$, L. Peters ${ }^{1}$, J. Peterson ${ }^{38}$, S. Philippen ${ }^{1}$, D. Pieloth ${ }^{23}$, S. Pieper ${ }^{58}$, M. Pittermann ${ }^{32}$, A. Pizzuto ${ }^{38}$, M. Plum ${ }^{40}$, Y. Popovych $^{39}$, A. Porcelli ${ }^{29}$, M. Prado Rodriguez ${ }^{38}$, P. B. Price ${ }^{8}$, B. Pries ${ }^{24}$, G. T. Przybylski ${ }^{9}$, C. Raab ${ }^{12}$, A. Raissi ${ }^{18}$, M. Rameez ${ }^{22}$, K. Rawlins $^{3}$, I. C. Rea ${ }^{27}$, A. Rehman ${ }^{42}$, P. Reichherzer ${ }^{11}$, R. Reimann ${ }^{1}$, G. Renzi ${ }^{12}$, E. Resconi ${ }^{27}$, S. Reusch ${ }^{59}$, W. Rhode ${ }^{23}$, M. Richman ${ }^{45}$, B. Riedel ${ }^{38}$, E. J. Roberts ${ }^{2}$, S. Robertson ${ }^{8,9}$, G. Roellinghoff ${ }^{52}$, M. Rongen ${ }^{39}$, C. Rott ${ }^{49}, 52$, T. Ruhe ${ }^{23}$, D. Ryckbosch ${ }^{29}$, D. Rysewyk $\mathrm{Cantu}^{24}$, I. Safa ${ }^{14,38}$, J. Saffer ${ }^{32}$, S. E. Sanchez Herrera ${ }^{24}$, A. Sandrock ${ }^{23}$, J. Sandroos ${ }^{39}$, M. Santander ${ }^{54}$, S. Sarkar ${ }^{44}$, S. Sarkar ${ }^{25}$, K. Satalecka $^{59}$, M. Scharf ${ }^{1}$, M. Schaufel ${ }^{1}$, H. Schieler ${ }^{31}$, S. Schindler ${ }^{26}$, P. Schlunder ${ }^{23}$, T. Schmidt ${ }^{19}$, A. Schneider ${ }^{38}$, J. Schneider ${ }^{26}$, F. G. Schröder ${ }^{31,42}$, L. Schumacher ${ }^{27}$, G. Schwefer ${ }^{1}$, S. Sclafani ${ }^{45}$, D. Seckel ${ }^{42}$, S. Seunarine ${ }^{47}$, A. Sharma ${ }^{57}$, S. Shefali $^{32}$, M. Silva $^{38}$, B. Skrzypek ${ }^{14}$, B. Smithers ${ }^{4}$, R. Snihur ${ }^{38}$, J. Soedingrekso ${ }^{23}$, D. Soldin $^{42}$, C. Spannfellner ${ }^{27}$, G. M. Spiczak ${ }^{47}$, C. Spiering ${ }^{59,}$, ${ }^{2}$, J. Stachurska $^{59}$, M. Stamatikos ${ }^{21}$, T. Stanev ${ }^{42}$, R. Stein ${ }^{59}$, J. Stettner ${ }^{1}$, A. Steuer ${ }^{39}$, T. Stezelberger ${ }^{9}$, T. Stürwald ${ }^{58}$, T. Stuttard ${ }^{22}$, G. W. Sullivan $^{19}$, I. Taboada ${ }^{6}$, F. Tenholt ${ }^{11}$, S. Ter-Antonyan ${ }^{7}$, S. Tilav ${ }^{42}$, F. Tischbein ${ }^{1}$, K. Tollefson ${ }^{24}$, L. Tomankova ${ }^{11}$, C. Tönnis ${ }^{53}$, S.

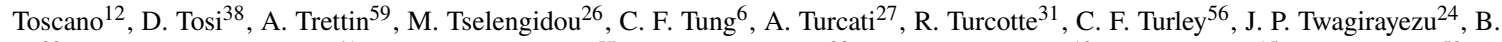
$\mathrm{Ty}^{38}$, M. A. Unland Elorrieta ${ }^{41}$, N. Valtonen-Mattila ${ }^{57}$, J. Vandenbroucke ${ }^{38}$, N. van Eijndhoven ${ }^{13}$, D. Vannerom ${ }^{15}$, J. van Santen $^{59}, \mathrm{~S}$. Verpoest $^{29}$, M. Vraeghe ${ }^{29}$, C. Walck ${ }^{50}$, T. B. Watson ${ }^{4}$, C. Weaver ${ }^{24}$, P. Weigel ${ }^{15}$, A. Weindl ${ }^{31}$, M. J. Weiss ${ }^{56}$, J. Weldert $^{39}$, C. Wendt $^{38}$, J. Werthebach ${ }^{23}$, M. Weyrauch ${ }^{32}$, N. Whitehorn ${ }^{24,35}$, C. H. Wiebusch ${ }^{1}$, D. R. Williams ${ }^{54}$, M. Wolf ${ }^{27}$, K. Woschnagg ${ }^{8}$, G. Wrede ${ }^{26}$, J.

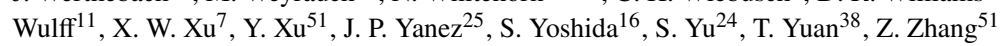

${ }^{1}$ III. Physikalisches Institut, RWTH Aachen University, D-52056 Aachen, Germany

2 Department of Physics, University of Adelaide, Adelaide, 5005, Australia

${ }^{3}$ Dept. of Physics and Astronomy, University of Alaska Anchorage, 3211 Providence Dr., Anchorage, AK 99508, USA

${ }^{4}$ Dept. of Physics, University of Texas at Arlington, 502 Yates St., Science Hall Rm 108, Box 19059, Arlington, TX 76019, USA

${ }^{5}$ CTSPS, Clark-Atlanta University, Atlanta, GA 30314, USA

${ }^{6}$ School of Physics and Center for Relativistic Astrophysics, Georgia Institute of Technology, Atlanta, GA 30332, USA

${ }^{7}$ Dept. of Physics, Southern University, Baton Rouge, LA 70813, USA

${ }^{8}$ Dept. of Physics, University of California, Berkeley, CA 94720, USA

${ }^{9}$ Lawrence Berkeley National Laboratory, Berkeley, CA 94720, USA

${ }^{10}$ Institut für Physik, Humboldt-Universität zu Berlin, D-12489 Berlin, Germany

${ }^{11}$ Fakultät für Physik \& Astronomie, Ruhr-Universität Bochum, D-44780 Bochum, Germany

12 Université Libre de Bruxelles, Science Faculty CP230, B-1050 Brussels, Belgium

13 Vrije Universiteit Brussel (VUB), Dienst ELEM, B-1050 Brussels, Belgium

14 Department of Physics and Laboratory for Particle Physics and Cosmology, Harvard University, Cambridge, MA 02138, USA

15 Dept. of Physics, Massachusetts Institute of Technology, Cambridge, MA 02139, USA 
${ }^{16}$ Dept. of Physics and Institute for Global Prominent Research, Chiba University, Chiba 263-8522, Japan

${ }^{17}$ Department of Physics, Loyola University Chicago, Chicago, IL 60660, USA

${ }^{18}$ Dept. of Physics and Astronomy, University of Canterbury, Private Bag 4800, Christchurch, New Zealand

${ }^{19}$ Dept. of Physics, University of Maryland, College Park, MD 20742, USA

${ }^{20}$ Dept. of Astronomy, Ohio State University, Columbus, OH 43210, USA

${ }^{21}$ Dept. of Physics and Center for Cosmology and Astro-Particle Physics, Ohio State University, Columbus, OH 43210, USA

${ }^{22}$ Niels Bohr Institute, University of Copenhagen, DK-2100 Copenhagen, Denmark

${ }^{23}$ Dept. of Physics, TU Dortmund University, D-44221 Dortmund, Germany

24 Dept. of Physics and Astronomy, Michigan State University, East Lansing, MI 48824, USA

${ }^{25}$ Dept. of Physics, University of Alberta, Edmonton, Alberta, Canada T6G 2E1

${ }^{26}$ Erlangen Centre for Astroparticle Physics, Friedrich-Alexander-Universität Erlangen-Nürnberg, D-91058 Erlangen, Germany

${ }^{27}$ Physik-department, Technische Universität München, D-85748 Garching, Germany

${ }^{28}$ Département de physique nucléaire et corpusculaire, Université de Genève, CH-1211 Genève, Switzerland

${ }^{29}$ Dept. of Physics and Astronomy, University of Gent, B-9000 Gent, Belgium

${ }^{30}$ Dept. of Physics and Astronomy, University of California, Irvine, CA 92697, USA

${ }^{31}$ Karlsruhe Institute of Technology, Institute for Astroparticle Physics, D-76021 Karlsruhe, Germany

${ }^{32}$ Karlsruhe Institute of Technology, Institute of Experimental Particle Physics, D-76021 Karlsruhe, Germany

${ }^{3}$ Dept. of Physics, Engineering Physics, and Astronomy, Queen's University, Kingston, ON K7L 3N6, Canada

${ }^{34}$ Dept. of Physics and Astronomy, University of Kansas, Lawrence, KS 66045, USA

${ }^{35}$ Department of Physics and Astronomy, UCLA, Los Angeles, CA 90095, USA

${ }^{36}$ Department of Physics, Mercer University, Macon, GA 31207-0001, USA

${ }^{37}$ Dept. of Astronomy, University of Wisconsin-Madison, Madison, WI 53706, USA

${ }^{38}$ Dept. of Physics and Wisconsin IceCube Particle Astrophysics Center, University of Wisconsin-Madison, Madison, WI 53706, USA

${ }^{39}$ Institute of Physics, University of Mainz, Staudinger Weg 7, D-55099 Mainz, Germany

${ }^{40}$ Department of Physics, Marquette University, Milwaukee, WI, 53201, USA

${ }^{41}$ Institut für Kernphysik, Westfälische Wilhelms-Universität Münster, D-48149 Münster, Germany

42 Bartol Research Institute and Dept. of Physics and Astronomy, University of Delaware, Newark, DE 19716, USA

${ }^{43}$ Dept. of Physics, Yale University, New Haven, CT 06520, USA

44 Dept. of Physics, University of Oxford, Parks Road, Oxford OX1 3PU, UK

${ }^{45}$ Dept. of Physics, Drexel University, 3141 Chestnut Street, Philadelphia, PA 19104, USA

${ }^{46}$ Physics Department, South Dakota School of Mines and Technology, Rapid City, SD 57701, USA

${ }^{47}$ Dept. of Physics, University of Wisconsin, River Falls, WI 54022, USA

${ }^{48}$ Dept. of Physics and Astronomy, University of Rochester, Rochester, NY 14627, USA

${ }^{49}$ Department of Physics and Astronomy, University of Utah, Salt Lake City, UT 84112, USA

${ }^{50}$ Oskar Klein Centre and Dept. of Physics, Stockholm University, SE-10691 Stockholm, Sweden

${ }^{51}$ Dept. of Physics and Astronomy, Stony Brook University, Stony Brook, NY 11794-3800, USA

52 Dept. of Physics, Sungkyunkwan University, Suwon 16419, Korea

${ }^{53}$ Institute of Basic Science, Sungkyunkwan University, Suwon 16419, Korea

${ }^{54}$ Dept. of Physics and Astronomy, University of Alabama, Tuscaloosa, AL 35487, USA

${ }^{55}$ Dept. of Astronomy and Astrophysics, Pennsylvania State University, University Park, PA 16802, USA

${ }^{56}$ Dept. of Physics, Pennsylvania State University, University Park, PA 16802, USA

57 Dept. of Physics and Astronomy, Uppsala University, Box 516, S-75120 Uppsala, Sweden

${ }^{58}$ Dept. of Physics, University of Wuppertal, D-42119 Wuppertal, Germany

${ }^{59}$ DESY, D-15738 Zeuthen, Germany

${ }^{60}$ Università di Padova, I-35131 Padova, Italy

${ }^{61}$ National Research Nuclear University, Moscow Engineering Physics Institute (MEPhI), Moscow 115409, Russia

${ }^{62}$ Earthquake Research Institute, University of Tokyo, Bunkyo, Tokyo 113-0032, Japan

\section{Acknowledgements}

USA - U.S. National Science Foundation-Office of Polar Programs, U.S. National Science Foundation-Physics Division, U.S. National Science Foundation-EPSCoR, Wisconsin Alumni Research Foundation, Center for High Throughput Computing (CHTC) at the University of Wisconsin-Madison, Open Science Grid (OSG), Extreme Science and Engineering Discovery Environment (XSEDE), Frontera computing project at the Texas Advanced Computing Center, U.S. Department of Energy-National Energy Research Scientific Computing Center, Particle astrophysics research computing center at the University of Maryland, Institute for Cyber-Enabled Research at Michigan State University, and Astroparticle physics computational facility at Marquette University; Belgium - Funds for Scientific Research (FRS-FNRS and FWO), FWO Odysseus and Big Science programmes, and Belgian Federal Science Policy Office (Belspo); Germany - Bundesministerium für Bildung und Forschung (BMBF), Deutsche Forschungsgemeinschaft (DFG), Helmholtz Alliance for Astroparticle Physics (HAP), Initiative and Networking Fund of the Helmholtz Association, Deutsches Elektronen Synchrotron (DESY), and High Performance Computing cluster of the RWTH Aachen; Sweden - Swedish Research Council, Swedish Polar Research Secretariat, Swedish National Infrastructure for Computing (SNIC), and Knut and Alice Wallenberg Foundation; Australia - Australian 
Research Council; Canada - Natural Sciences and Engineering Research Council of Canada, Calcul Québec, Compute Ontario, Canada Foundation for Innovation, WestGrid, and Compute Canada; Denmark - Villum Fonden and Carlsberg Foundation; New Zealand Marsden Fund; Japan - Japan Society for Promotion of Science (JSPS) and Institute for Global Prominent Research (IGPR) of Chiba University; Korea - National Research Foundation of Korea (NRF); Switzerland - Swiss National Science Foundation (SNSF); United Kingdom - Department of Physics, University of Oxford. 